\title{
Successful Carotid Stenting for Chronic Total Occlusion of the Internal Carotid Artery
}

\author{
Won Ho Kim, MD' ${ }^{1}$, Pil-Ki Min, $\mathrm{MD}^{3}$, Dong Jun Kim, $\mathrm{MD}^{2}$ and Won-Heum Shim, MD \\ ${ }^{I}$ Division of Cardiology, Yonsei Cardiovascular Hospital, ${ }^{2}$ Division of Radiology, Severance Hospital, \\ ${ }^{3}$ Division of Cardiology, Gangnam Severance Hospital, Yonsei University College of Medicine, Seoul, Korea
}

\section{ABSTRACT}

A 64-year-old man complaining of pulsatile headache was admitted. Imaging studies revealed a near-total occlusion of the right proximal internal carotid artery (ICA) with slow antegrade flow into the distal ICA. Right cerebral flow was supplied by collateral flow through the posterior communicating and ophthalmic arteries. He was successfully treated by carotid artery stenting. No new neurological deficit or transient ischemic attack occurred after treatment. (Korean Circ J 2010;40:288-291)

KEY WORDS: Thrombosis, internal carotid artery; Stents.

\section{Introduction}

The development of endovascular therapy has enabled recanalization of chronic totally occluded coronary and subclavian arteries. ${ }^{1)}$ However, little is known about the feasibility and safety of endovascular treatment for chronic total occlusion (CTO) of the carotid artery. We describe a case of successful stenting for a CTO of the right internal carotid artery (ICA) with cerebral hypoperfusion.

\section{Case}

\section{History and pre-intervention evaluation}

A 64-year-old man was admitted complaining of pulsatile headache. The patient had a history of coronary artery disease and a cerebrovascular accident 2 years ago. He was ex-smoker. There was no history of diabetes, arrhythmia, or cardiac valvular disease. His blood pressure was normal. Auscultation of the neck showed normal

\section{Received: September 29, 2009}

Accepted: October 26, 2009

Correspondence: Won-Heum Shim, MD, Division of Cardiology, Yonsei Cardiovascular Hospital, Yonsei University College of Medicine, 250 Seongsanro, Seodaemun-gu, Seoul 120-752, Korea

Tel: 82-2-2228-8457, Fax: 82-2-393-2041

E-mail:whshim@yuhs.ac

(c) This is an Open Access article distributed under the terms of the Creative Commons Attribution Non-Commercial License (http://creativecommons. org/licenses/by-nc/3.0) which permits unrestricted non-commercial use, distribution, and reproduction in any medium, provided the original work is properly cited. carotid upstrokes. He was alert and oriented. A detailed neurologic examination was normal. An electrocardiogram showed a normal sinus rhythm. An echocardiogram showed no cardiac embolic source.

Magnetic resonance (MR) imaging of the brain and neck showed old ischemic lesions in the bilateral centrum semiovale, left basal ganglia, and a CTO of the right ICA. Diagnostic cerebral angiograms showed a CTO of the right proximal ICA with slow antegrade flow into the distal ICA. Right cerebral flow was supplied by collateral flow through the posterior communicating and ophthalmic arteries (Fig. 1). Single-photon emission CT (SPECT) scanning with ${ }^{99 \mathrm{~m}} \mathrm{Tc}$-ethyl cysteinate dimmer revealed decreased uptake in the right cerebral hemisphere with reduced reactivity to the Diamox challenge test, suggestive of a decrease in vascular reserve (Fig. 2). Angioplasty was risky because there were multiple, calcified long lesions, and stenting was technically difficult. We proposed extracranial-intracranial (EC-IC) bypass surgery, but he refused surgery. The patient suffered from pulsatile headache and it was refractory to medical treatment. Imaging studies showed definite evidence of ischemia, and the collateral flow through the posterior communicating and ophthalmic arteries was insufficient. Judging from symptoms, imaging studies, and a history of CVA, we thought that endovascular recanalization was required.

\section{Endovascular procedure}

A 6 French $(\mathrm{F})$ sheath was introduced into the right femoral artery. A 5F diagnostic catheter was placed in 


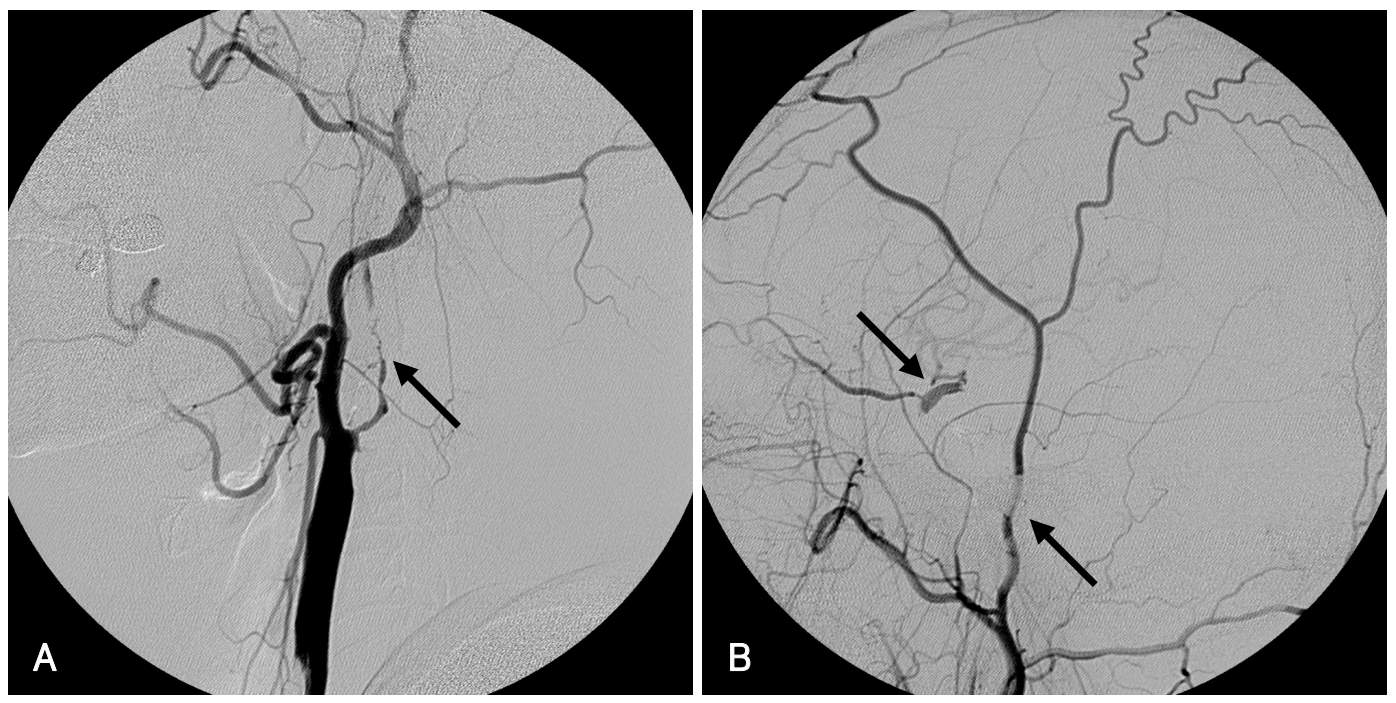

Fig. 1. A: cerebral angiography from the right CCA confirms the presence of a near-total occlusion of the right proximal ICA with slow antegrade flow into the distal ICA (arrow). B: right cerebral flow is supplied by collateral flow through the posterior communicating (arrow) and ophthalmic arteries (arrow), as shown on an angiogram of the extracranial artery. CCA: common carotid artery, ICA: internal carotid artery.

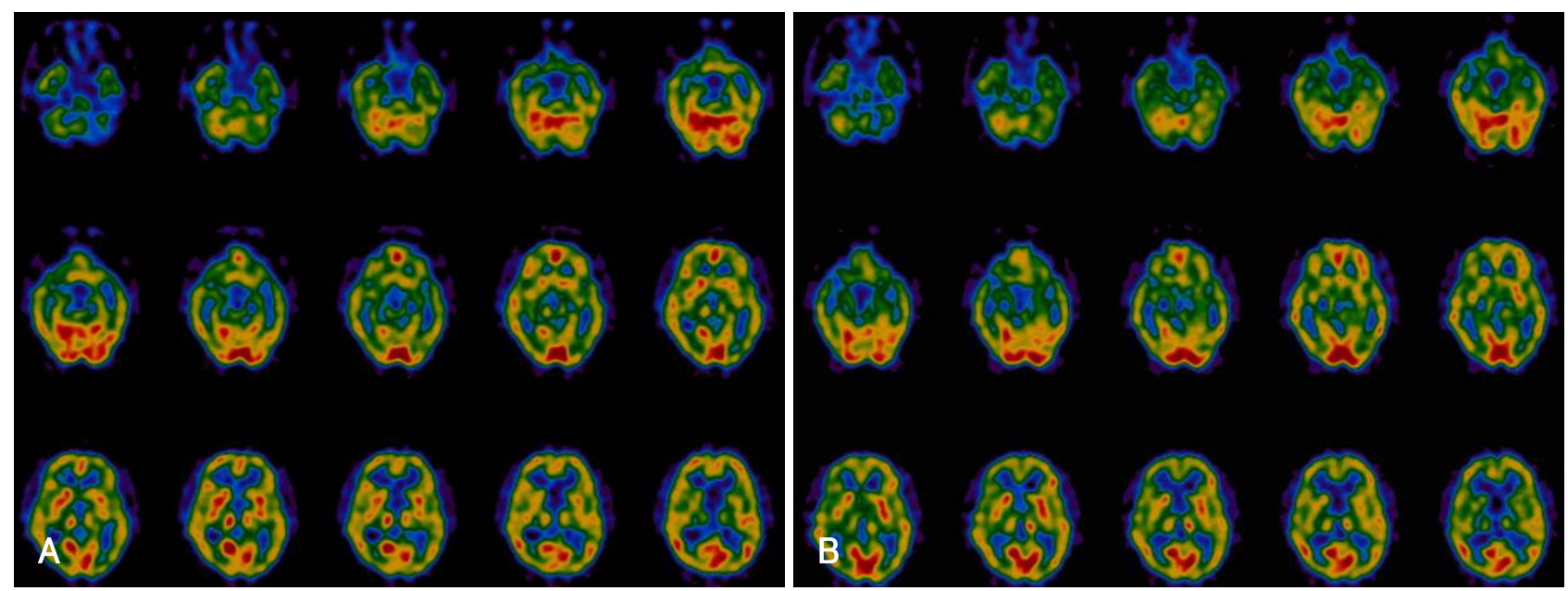

Fig. 2. A: single-photon emission CT scanning with a ${ }^{99 m}$ Tc-ethyl cysteinate dimmer revealed decreased uptake in the right cerebral hemisphere. B: scans obtained after the Diamox challenge showed no increase in cerebral flow in the right cerebral hemisphere, suggestive of a decrease in vascular reserve.

the right innominate artery and a 0.035-inch guidewire (Terumo Corp., Tokyo, Japan) was used to exchange a 7F Shuttle-SL guide sheath (Cook, Bloomington, IN, USA) into the right common carotid artery (CCA). Heparinization was performed during the intervention with the active clotting time being kept at about 250 to 300 seconds. The 7F Shuttle-SL guide sheath was positioned proximal to the right common carotid bifurcation. A microcatheter was placed proximal to the occlusion to improve the backup support for the wire manipulation. While avoiding excessive rotational or drilling motion of the wire, successive small penetrate-and-advance steps were made along the imaginary tract of the occluded vessel segment. The lesion was crossed with a 0.014-inch guidewire, the tip of which was positioned in the middle cerebral artery. The microcatheter was exchanged for a $1.25 \times 10 \mathrm{~mm}$ balloon, which was inflated to predilate the occlusion from the distal portion of the ICA initially and moved to the proximal portion of the ICA. An embolic protection device could not be used because the stenosis was very hard and the manipulation of the device was technically difficult. Follow-up angiography showed reduction of the stenosis and insufficient revascularization of the ICA. The catheter was exchanged for a $1.5 \times 20 \mathrm{~mm}$ balloon, and the ICA was dilated in the same manner. Next, balloon angioplasty with a $2.5 \times 30$ $\mathrm{mm}$ balloon was performed at the bifurcation of the CCA. After successive balloon angioplasty, the angiogram showed a dilated and patent ICA with narrowing at the bifurcation. A $9 \times 50 \mathrm{~mm}$ self-expanding stent (Carotid Wallstent, Boston Scientific, Galway, Ireland) was placed to cover the occluded portion, followed by post-dilation to achieve a residual diameter stenosis of $<20 \%$ (Fig. 3). The final angiogram showed patency of 

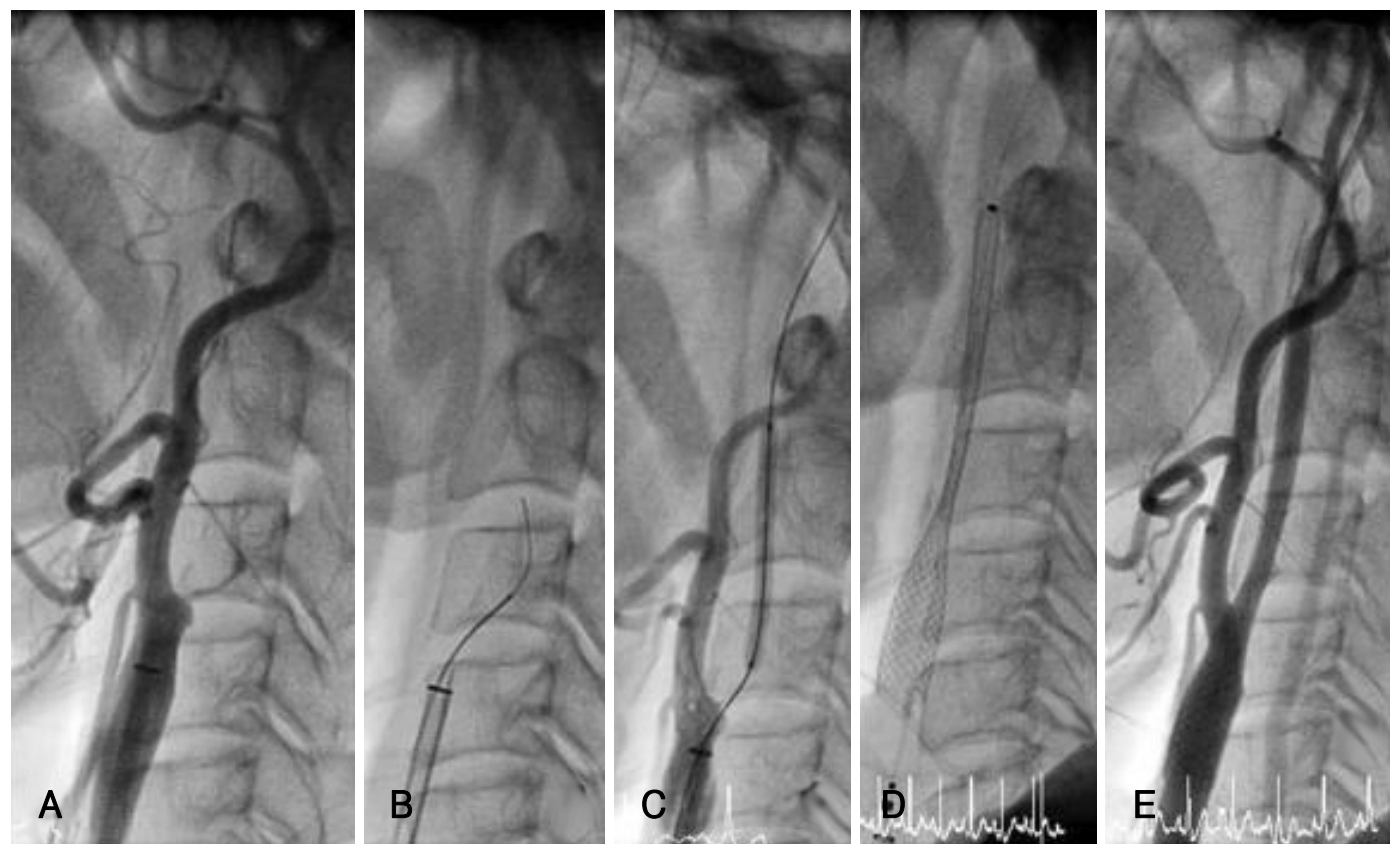

Fig. 3. Endovascular treatment. A: proximal ICA occlusion. B: the wire advancing with the microcatheter. C: predilatation with a coronary balloon. D: successful stenting insertion. E: final angiogram after carotid stent implantation after adjuvant balloon post-dilation.
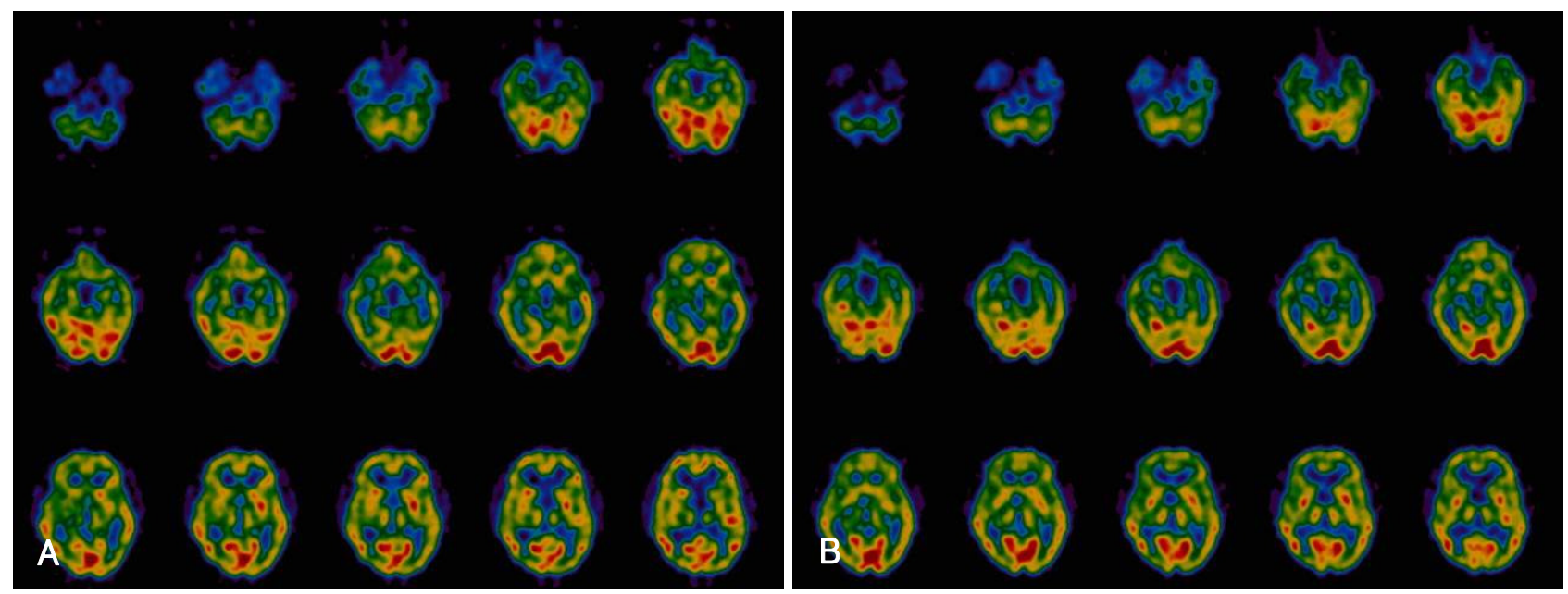

Fig. 4. Single-photon emission CT scanning with a ${ }^{99 \mathrm{~m}}$ Tc-ethyl cysteinate dimmer obtained after endovascular recanalization. A: cerebral blood flow at rest increased in the right frontotemporal region compared to that before treatment. B: vascular reactivity for the Diamox challenge also recovered.

the carotid artery, with re-established antegrade filling of the middle and anterior cerebral arteries. The reversed ophthalmic artery flow was normalized.

The patient had no neurologic changes following the procedure. Follow up SPECT revealed an increase in cerebral blood flow with recovery of vascular reactivity (Fig. 4). Also, MR angiography revealed complete recanalization of the right ICA. At 30-day follow up (Fig. 5), he continued to be free of symptoms.

\section{Discussion}

There is a direct relationship between the degree of carotid artery stenosis/occlusion and the risk of ipsila- teral stroke. ${ }^{1-4)}$ Carotid artery stenting has emerged as an alternative treatment to carotid endarterectomy for this common disorder. ${ }^{5-7)}$ The SAPPHIRE investigators reported that carotid stenting was not inferior to carotid endarterectomy for the treatment of carotid artery stenosis at 3 years. ${ }^{8)}$

However, CTO of the carotid artery is different. Endarterectomy prevents stroke in patients with ICA stenosis, but the success rate in recanalizing occlusions is as low as $34 \%$ because of technical difficulties. ${ }^{9)}$ Surgical bypass may be a natural resolution for ICA occlusion, although the large international randomized ECIC bypass trial failed to show any benefit. ${ }^{10)}$ Recently, there have been few reports of revascularization by CTO 

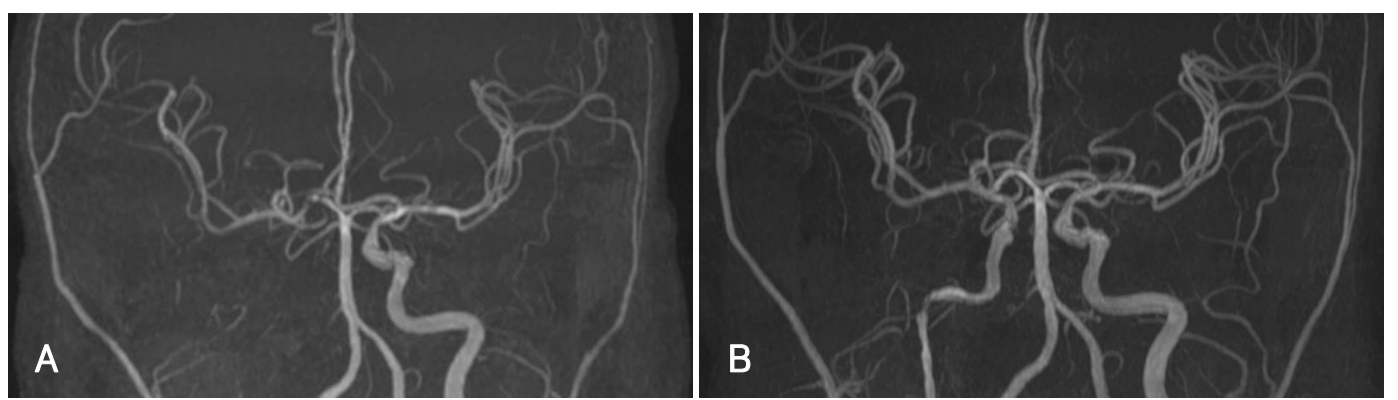

Fig. 5. Post-procedural magnetic resonance angiography revealed complete recanalization of the right ICA with normal antegrade flow distal to the previously occluded segment $(B)$ compared to pre-procedural magnetic resonance angiography (A).

of the ICA by percutaneous angioplasty. Terada et al. ${ }^{1)}$ reported the use of angioplasty for a CTO of the cervical ICA with cerebral hypoperfusion. Their study showed that endovascular recanalization can be performed for symptomatic ICA occlusion, even in the chronic stage of the illness. Komiyama et al. ${ }^{11)}$ demonstrated that percutaneous angioplasty for a CTO of the intracranial ICA is technically feasible and can be an alternative to ECIC bypass surgery in a selected group of patients with symptomatic hemodynamic compromise.

Cerebral embolization occurs during all stages of carotid artery stenting. Embolic protection devices (EPDs) reduced the stroke risk involved with carotid artery stenting in analysis of the Global Carotid Artery Stent Registry. The incidence of death/stroke was $6.93 \%$ without use of EPDs, but was 3.22\% with EPDs. ${ }^{12)}$ Despite this efficacy, we could not use an EPD because of technical problems.

The overall rate of subsequent stroke is $7 \%$ per year and $5.9 \%$ per year for ischemic stroke ipsilateral to the CTO of the ICA. ${ }^{13)}$ These risks are higher in patients with hemodynamic impairment. ${ }^{14)}$ Recently, Kao et al. ${ }^{15)}$ demonstrated that stenting for cervical ICA occlusion is feasible with acceptable midterm clinical results. Although this is a single case report, our case also suggests that endovascular revascularization can be performed in some patients with chronic ICA occlusions.

\section{Conclusions}

Our case shows that carotid stenting can be a successful alternative to EC-IC bypass surgery in patients with CTO of the ICA.

\section{REFERENCES}

1) Terada T, Yamaga H, Tsumoto T, Masuo O, Itakura T. Use of an embolic protection system during endovascular recanalization of a totally occluded cervical internal carotid artery at the chronic stage: case report. J Neurosurg 2005; 102:558-64.
2) Rothwell PM, Eliasziw M, Gutnikov SA, et al. Analysis of pooled data from the randomised controlled trials of endarterectomy for symptomatic carotid stenosis. Lancet 2003;361:107-16.

3) Inzitari D, Eliasziw M, Gates P, et al. The causes and risk of stroke in patients with asymptomatic internal-carotid-artery stenosis. $N$ Engl J Med 2000;342:1693-700.

4) Ohki T, Parodi J, Veith FJ, et al. Efficacy of a proximal occlusion catheter with reversal of flow in the prevention of embolic events during carotid artery stenting: an experimental analysis. $J$ Vasc Surg 2001;33:504-9.

5) European Carotid Surgery Trial. Randomised trial of endarterectomy for recently symptomatic carotid stenosis: final results of the MRC European Carotid Surgery Trial (ECST). Lancet 1998; 351:1379-87.

6) Roubin GS, New G, Iyer SS, et al. Immediate and late clinical outcomes of carotid artery stenting in patients with symptomatic and asymptomatic carotid artery stenosis: a 5-year prospective analysis. Circulation 2001;103:532-7.

7) Ko YG, Park SH, Kim JY, et al. Carotid artery stenting with distal protection device: early experience. Korean Circ J 2005;35: 61-8.

8) Gurm HS, Yadav JS, Fayad P, et al. Long-term results of carotid stenting versus endarterectomy in high-risk patients. $N$ Engl J Med 2008;358:1572-9.

9) Paty PSK, Adeniyi JA, Mehta M, et al. Surgical treatment of internal carotid artery occlusion. J Vasc Surg 2003;37:785-8.

10) The EC-IC Bypass Study Group. Failure of extracranial-intracranial arterial bypass to reduce the risk of ischemic stroke: results of an international randomized trial. N Engl J Med 1985;313: $1191-200$.

11) Komiyama M, Yoshimura M, Honnda Y, Matsusaka Y, Yasui Y. Percutaneous angioplasty of a chronic total occlusion of the intracranial internal carotid artery: case report. Surg Neurol 2006; 66:513-8.

12) Dieter RS, Laird JR. Carotid artery stenting: update. Int J Cardiovasc Intervent 2005; 7:126-33.

13) Hankey GJ, Warlow CP. Prognosis of symptomatic carotid occlusion: an overview. Cerebrovasc Dis 1991;1:245-56.

14) Derdeyn CP, Grubb RL Jr, Powers WJ. Cerebral hemodynamic impairment: methods of measurement and association with stroke risk. Neurology 1999;53:251-9.

15) Kao HL, Lin MS, Wang CS, et al. Feasibility of endovascular recanalization for symptomatic cervical internal carotid artery occlusion. J Am Coll Cardiol 2007;49:765-71. 